\title{
Steganographic Scheme Based on Message-Cover matching
}

\author{
Youssef Taouil and El Bachir Ameur \\ Department of Computer Sciences, Faculty of Sciences, University Ibn Tofail, Morocco
}

\begin{tabular}{l}
\hline \hline Article Info \\
\hline Article history: \\
Received November 15, 2017 \\
Revised May 25, 2018 \\
Accepted July 1, 2018
\end{tabular}

\section{Keyword:}

Steganography

Data hiding

Permutation

Least significant bit

Faber-Schauder DWT

\begin{abstract}
Steganography is one of the techniques that enter into the field of information security, it is the art of dissimulating data into digital files in an imperceptible way that does not arise the suspicion. In this paper, a steganographic method based on the FaberSchauder discrete wavelet transform is proposed. The embedding of the secret data is performed in Least Significant Bit $(L S B)$ of the integer part of the wavelet coefficients. The secret message is decomposed into pairs of bits, then each pair is transformed into another based on a permutation that allows to obtain the most matches possible between the message and the $L S B$ of the coefficients. To assess the performance of the proposed method, experiments were carried out on a large set of images, and a comparison to prior works is accomplished. Results show a good level of imperceptibility and a good trade-off imperceptibility-capacity compared to literature.
\end{abstract}

Copyright (C) 2018 Institute of Advanced Engineering and Science. All rights reserved.

\section{Corresponding Author:}

Youssef Taouil

LaRIT Laboratory, Department of Computer Sciences,

Faculty of Sciences, Ibn Tofail University,

14000 Kenitra, Morocco.

taouilysf@gmail.com

\section{INTRODUCTION}

Among the grounds discussed in the field of information security is the cryptography and data hiding. Cryptography protects information by coding its content to become incomprehensible to unauthorized people. But, even an incomprehensible message may attract the attention of eavesdroppers. To overcome this hindrance, steganography offers the aspect of "secrecy" rather than "incomprehensibility", it is the technique of concealing information through digital media. Its main objective is the secrecy of the information's existence so that no one aside the authorized recipient may suspect it. In steganography, various media files have been utilized as cover file such as audio, image, video and plain text, but among these media, the most popular one to dissimulate secret information is image since it is shared everyday on networks and also because the human visual system can not detect slight changes done on the image intensity.

Generally, there are two approaches of steganography: the spatial domain and the frequency domain. In the spatial domain approach, data is hidden directly in the pixels of the cover image, such as the Least Significant Bit $(L S B)$ Substitution" [1], [2]. However, this technique is vulnerable to statistical attacks, to protect the hidden message, authors in [3] proposed to encode it using the Hungarian puzzle. There is also the interpolation based techniques [4], [5], [6] where data is hidden in the error between the initial pixels and the interpolated pixels, and the Pixel Value Differencing (PVD) [7], [8], [9] where data is hidden in the difference between each neighbouring pixels. In [10], authors proposed a steganographic algorithm based on Ant Colony Optimization (ACO), the ACO algorithm is used to select the complex regions of the cover image, then data is hidden using the LSB substitution in the selected areas.

In the frequency domain based techniques, data is hidden in the coefficients of the transform domain, such as Discrete Cosinus Transform $(D C T)$ and Discrete Wavelet Transform (DWT). In [11], a reversible data hiding for JPEG images is proposed, the quantization table is modified through dividing some of its elements by an integer while multiplying the corresponding quantized $D C T$ coefficients by the same integer in order to create space for the dissimulation. In [12], authors proposed a steganographic scheme for JPEG that preserves 
the $D C T$ coefficients histogram in order to resist steganalysis based attacks. The scheme distinguishes sensitive pixels and protects them from the extra bit embedding to reduce the distortions in the histogram. In [13], the Integer Wavelet Transform (IWT) is applied to each $8 \times 8$ blocks of the cover image, then the zero tree method is utilized to select the proper location where data can be embedded. In [14], a steganographic scheme based on the Haar DWT is proposed, data is hidden in the first $L S B$ of the DWT coefficients, the algorithm is generalized on $K-L S B$ with the use of the Optima Pixel Adjustment $O P A$ procedure in [15]. In [16], the edge IWT coefficients are classified based on their Most Significant bit (MSB), the size of data to be hidden in the coefficient is determined based on the value of the coefficient's MSBs.

In this paper, we propose a steganographic scheme based on the Faber Schauder DWT, this transform allows us to hide data in the integer part without worrying about the problem of the floating point (the pixels of the stego image are guaranteed to be integers). Data is hidden in the $L S B$ s of the transform details. The message and the coefficients $L S B s$ are decomposed to pairs of bits $\bar{m}_{k}$ and $\bar{z}_{k}$, and based on the matrix that illustrates the difference of distance between $\bar{m}_{k}$ and $\bar{z}_{k}$, we search for the permutation that transforms the message into the binary sequence that has the most possible matches with $\bar{z}_{k}$. The selection order of the coefficients where to dissimulate data is given aleatory by a random key. Experiments were performed on a large set of a variety of images to assess the perfomance of the proposed work, and comparison to prior works is accomplished. Results indicate good level of imperceptibility and trade-off capacity-imperceptibility.

The remaining of the paper is organized as follows: Section 2 details the algorithms of decomposition and reconstruction of the Faber-Schauder DWT. In section 3, the proposed steganography method is explained. In section 4, experimental results of the test and comparison are discussed. Section 5 concludes the paper.

\section{FABER-SCHAUDER DWT}

The Faber-Schauder Wavelet transform is a multi-scale transform, the multi-scale analysis is formulated based on the study of compactly supported wavelet bases, it is the main theory in wavelets that analyzes in detail a signal in the frequency domain. Multi-Scale Analysis of $L^{2}(\mathbb{R})$ is a sequence of nested vector spaces $\left(V_{j}\right)_{j \in \mathbb{Z}}\left(\cdots \subset V_{j+2} \subset V_{j+1} \subset V_{j} \subset V_{j-1} \subset \cdots\right)$.

For all $j$ in $\mathbb{Z}, V_{j+1} \subset V_{j}$. Let $W_{j+1}$ be a supplementary of $V_{j+1}$ in $V_{j}:\left(V_{j}=V_{j+1} \oplus W_{j+1}\right)$, the basis of Faber-Schauder is the basis of $W_{j+1}$ given by the family of functions $\left(\psi_{n}^{j}\right)_{n \in \mathbb{Z}}$ with: $\psi_{n}^{j}=\varphi_{2 n+1}^{j}$, where:

$$
\varphi_{n}^{j}(t)=2^{-j} \varphi\left(2^{-j} t-n\right), \text { and } \varphi(t)=\left\{\begin{array}{lll}
1+t & \text { if } & -1 \leq t \leq 0 \\
1-t & \text { if } & 0 \leq t \leq 1 \\
0 & \text { if } & t \notin[-1,1]
\end{array}\right.
$$

In two dimensions, $L^{2}\left(\mathbb{R}^{2}\right)$ is approximated by using the tensor product:

$$
\begin{gathered}
\tilde{V}_{j}=V_{j} \otimes V_{j}=\left(V_{j+1} \oplus W_{j+1}\right) \otimes\left(V_{j+1} \oplus W_{j+1}\right), \text { where: } \tilde{V}_{j+1}=V_{j+1} \otimes V_{j+1}, \text { and } \\
\tilde{W}_{j+1}=\left(V_{j+1} \otimes W_{j+1}\right)+\left(W_{j+1} \otimes V_{j+1}\right)+\left(W_{j+1} \otimes W_{j+1}\right)
\end{gathered}
$$

Thus, an image $C$ is decomposed into four blocks: $A, H, V$ and $D$ as illustrated in Fig. 1. A corresponds to $\tilde{V}_{j+1}$, while $H, V$, and $D$ correspond respectively to the three subspaces of $\tilde{W}_{j+1}$.

The decomposition algorithm of Faber-Schauder $D W T$ is given by the following equations:

$$
\left\{\begin{array}{l}
A(i, j)=C(2 i, 2 j) \\
H(i, j)=C(2 i+1,2 j)-\frac{C(2 i, 2 j)+C(2 i+2,2 j)}{2} \\
V(i, j)=C(2 i, 2 j+1)-\frac{C(2 i, 2 j)+C(2 i, 2 j+2)}{2} \\
D(i, j)=C(2 i+1,2 j+1)-\sum_{k=0}^{1} \sum_{r=0}^{1} \frac{C(2 i+2 k, 2 j+2 r)}{4}
\end{array}\right.
$$

The reconstruction algorithm or inverse Faber-Schauder Discrete Wavelet Transform is given by the 


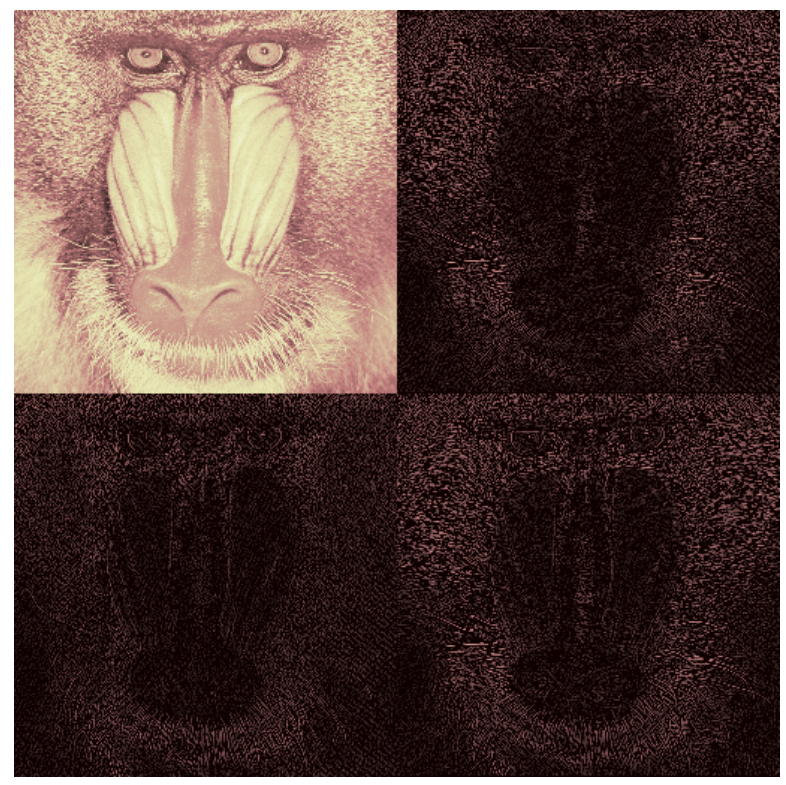

Figure 1. One level 2D Faber-Schauder DWT of the image Baboon

following equations:

$$
\begin{cases}C(2 i, 2 j) & =A(i, j) \\ C(2 i+1,2 j) & =H(i, j)-\frac{A(i, j)+A(i+1, j)}{2} \\ C(2 i, j+1) & =V(i, j)-\frac{A(i, j)+A(i, j+1)}{2} \\ C(2 i+1,2 j+1) & =D(i, j)-\sum_{k=0}^{1} \sum_{r=0}^{1} \frac{A(i+k, j+r)}{4}\end{cases}
$$

\section{PROPOSED WORK}

\subsection{Embedding Process}

After the dissimulation of the secret message, the cover image is modified, the principal objective of steganography is to minimize this modification so that the hiding does not arise the suspicion of eavesdroppers. The proposed method is based on Faber-Schauder DWT. The block $A$ represents the approximation of the image cover, it contains the low frequencies where the human eye is sensitive to slightest modifications. $A$ should remain unchanged. Therefore, data is hidden in the three remaining blocks $H, V$, and $D$.

Let $m$ be the binary sequence of the secret message $m=\left\{m_{1}, \ldots, m_{L}\right\} m_{k} \in\{0,1\}$, and let $Z$ be the set of the $L S B$ of the integer part of the coefficients of the blocks $H, V$ and $D$. We divide $m$ and $Z$ into pairs $m=\bigcup \bar{m}_{k}$ and $Z=\bigcup \bar{z}_{k}$, where $\bar{m}_{k}=\left\{m_{2 k-1}, m_{2 k}\right\}$ and $\bar{z}_{k}=\left\{z_{2 k-1}, z_{2 k}\right\} . \forall k \bar{m}_{k}$ and $\bar{z}_{k}$ are in the set $E=\{\{0,0\},\{0,1\},\{1,0\},\{1,1\}\}$.

When $\bar{m}_{k}$ is hidden into $\bar{z}_{k}$ there are three possibilities: they are identical $\bar{m}_{k}=\bar{z}_{k}$, conjugate $\bar{m}_{k}+\bar{z}_{k}=\{1,1\}$ or they have one bit different. To visualize all these possibilities, we introduce the matrix $G$ whose elements denote the number of times each pair $\bar{m}_{k}$ from $E$ encounters a pair $\bar{z}_{k}$ from $E$. $G$ is a 4 x 4 matrix, because $\operatorname{card}(E)=4$, its elements are integer between 0 and $L / 2, G \in \mathbb{M}_{4,4}(\{0, \ldots, L / 2\})$. The first column is associated to $\{0,0\}$, the second column to $\{0,1\}$, the third column to $\{1,0\}$ and the last column to $\{1,1\}$. The same thing goes for the rows. If $\bar{m}_{k}$ encounters $\bar{z}_{k}$, then the element $G_{i, j}$ from the $i$ row and $j$ column is incremented, where the relation between the pairs, rows and columns is given by the following function $f$ which is a bijection that associates each pair to its decimal value plus 1 : 


$$
\begin{aligned}
& j=f\left(m_{2 k-1}, m_{2 k}\right)=2 m_{2 k-1}+m_{2 k}+1 \\
& i=f\left(z_{2 k-1}, z_{2 k}\right)=2 z_{2 k-1}+z_{2 k}+1
\end{aligned}
$$

In each column, the diagonal element show how much times $\bar{m}_{k}$ and $\bar{z}_{k}$ are identical, the element of the $2 n d$ diagonal denotes the number of times when $\bar{m}_{k}$ and $\bar{z}_{k}$ are opposite or conjugate. The remaining two elements denote how much times $\bar{m}_{k}$ and $\bar{z}_{k}$ have on bit different.

Example: 26 denotes how many times $\{0,1\}$ of the message encounters $\{1,0\}$ in the the coefficients $L S B$ s.

$$
G=\left(\begin{array}{ccccc} 
& \{0,0\} & \{0,1\} & \{1,0\} & \{1,1\} \\
\{0,0\} & 64 & 36 & 87 & 51 \\
\{0,1\} & 38 & 57 & 72 & 93 \\
\{1,0\} & 19 & 26 & 18 & 17 \\
\{1,1\} & 29 & 16 & 27 & 21
\end{array}\right)
$$

The error generated by the dissimulation is expressed by the following expression:

$$
M S E=\frac{1}{M N} \sum_{i=0}^{M-1} \sum_{j=0}^{N-1}(S(i, j)-C(i, j))^{2}
$$

Let $H^{\prime}(i, j)$ be the coefficient produced after hiding data in $H(i, j)$, and $h(i, j)=H^{\prime}(i, j)-H(i, j)$ the difference coming from this dissimulation. We define $v(i, j)$ and $d(i, j)$ the same way. Therefore, using the reconstructions equations (1), the $M S E$ becomes:

$$
M S E=\frac{1}{M N} \sum_{i=0}^{M / 2-1} \sum_{j=0}^{N / 2-1} h(i, j)^{2}+v(i, j)^{2}+d(i, j)^{2}
$$

The diagonal elements of the matrix $G$ corresponds to when data is hidden with zero changes, the 2nd diagonal elements corresponds to when 2 changes are needed, and the rest corresponds to when one bit is changed to hide 2 bits, as described in the following matrix $W_{G}$.

$$
W_{G}=\left(\begin{array}{cccc}
\mathbf{0} & 1 & 1 & \mathbf{2} \\
1 & \mathbf{0} & \mathbf{2} & 1 \\
1 & \mathbf{2} & \mathbf{0} & 1 \\
\mathbf{2} & 1 & 1 & \mathbf{0}
\end{array}\right)
$$

Hence, we can reformulate the $M S E$ based on the matrix $G$ as follows:

$$
M S E=\frac{1}{M N} \sum_{i=1}^{4} \sum_{j=1}^{4} W_{G}(i, j) G(i, j)
$$

which we can reformulate as follows:

$$
M S E=\frac{1}{M N}\left(\sum_{i=1}^{4} \sum_{j=1}^{4} G_{i, j}+\sum_{i=1}^{4} G_{5-i, i}-\operatorname{tr}(G)\right)
$$

where $\operatorname{tr}(G)$ is the trace of the matrix $G: \operatorname{tr}(G)=\sum_{i=1}^{4} G_{i, i}$

Since $\sum_{i=1}^{4} \sum_{j=1}^{4} G_{i, j}$ is unchanged for all permutations, then we define the function $\vartheta$ which associates the remaining two terms to the permutation:

$$
\begin{aligned}
\vartheta: \mathbb{S}_{4} & \longrightarrow \mathbb{Z} \\
p & \longmapsto \sum_{i=1}^{4} G_{5-i, i}-\operatorname{tr}(G)
\end{aligned}
$$


To minimize the $M S E$, we calculate $\vartheta(p)$ for all possible $p$ in $\mathbb{S}_{4}$ (24 permutations). Then, we search for the permutation $p^{*}$ corresponding to the lowest value of $\vartheta(p) \cdot p^{*}=\min _{p \in \mathbb{S}_{4}}(\vartheta(p))$. The equation $p^{*}(j)=j^{\prime}$ signifies that the column $j$ is permuted into $j^{\prime}$, which means that the pair $\overline{m_{k}}$ associated to $j$ by the function $f$ introduced in (2) is consequently changed to the pair associated to $j^{\prime}$.

For example, if $p^{*}(3)=1$, then by using the function $f, f^{-1}(3)$ is changed into $f^{-1}(1)$ i.e. each pair $\{1,0\}$ in the secret message is changed into $\{0,0\}$. Hence, we obtain the transformation of the secret message $m^{\prime}$ that allows us to reach the lowest $M S E$ calculated. $m^{\prime}$ is given by:

$$
m^{\prime}=\bigcup_{k=1}^{L / 2} f^{-1} o p^{*} o f\left(\bar{m}_{k}\right)
$$

Example: We consider the message "HELLO", the binary sequence of this message is

$$
m=0100100001100101011011000110110001101111
$$

We decompose $m$ into pairs: $m=01|00| 10|00| 01|10| 01|01| 01|10| 11|00| 01|10| 11|00| 01|10| 11 \mid 11$. Suppose that the $L S B$ s of the coefficients are :

$$
Z=00|11| 00|01| 11|01| 10|00| 10|10| 10|11| 11|10| 00|10| 10|10| 00 \mid 10 .
$$

We construct the matrix $G$ :

$$
G=\left(\begin{array}{llll}
0 & 2 & 1 & 2 \\
1 & 0 & 1 & 0 \\
1 & 3 & 3 & 2 \\
2 & 2 & 0 & 0
\end{array}\right)
$$

The error now is 25 , which means that there is 25 among the 40 message bits that are going to be dissimulated into their opposite bits of the coefficients. Now, we calculate the errors of the 24 permutations and we choose the permutation $p^{*}$ associated to the lowest error. $p^{*}$ and its associated $G^{*}$ are given by

$$
p^{*}=\left(\begin{array}{cccc}
1 & 2 & 3 & 4 \\
4 & 3 & 2 & 1
\end{array}\right), \quad G^{*}=\left(\begin{array}{cccc}
2 & 1 & 2 & 0 \\
0 & 1 & 0 & 1 \\
2 & 3 & 3 & 1 \\
0 & 0 & 0 & 2
\end{array}\right)
$$

The error becomes 15 . Hence, we construct the new binary sequence $m^{\prime}$ based on the equation (3) as follows:

$$
\{0,0\} \rightarrow\{1,1\} \quad ; \quad\{0,1\} \rightarrow\{1,0\} \quad ; \quad\{1,0\} \rightarrow\{0,1\} \quad ; \quad\{1,1\} \rightarrow\{1,1\}
$$

\subsection{Extraction process}

In the extraction, we retrieve the message $m^{\prime}$ from the $L S B$ of the coefficients' integer part. To be able to obtain the actual message $m$, the permutation $p^{*}$ is needed. Thus, in the dissimulation phase, we hide an identifier in the first coefficients. $p^{*}(1), p^{*}(2), p^{*}(3)$ and $p^{*}(4)$ are hidden int the first eight coefficients. After the extraction of $m^{\prime}$, we use the $p^{*}(i)$ to retrieve the message $m$ as follows:

$$
m=\bigcup_{k=1}^{L / 2} f^{-1} o\left(p^{*}\right)^{-1} o f\left({\overline{m^{\prime}}}_{k}\right)
$$

where $\overline{m^{\prime}}{ }_{k}=\left\{m_{2 k}^{\prime}, m_{2 k+1}^{\prime}\right\}$ 


\section{Embedding algorithm}

- Read the cover image as two dimensional file.

- Perform the Faber-Schauder DWT.

- Construct the matrix $G$, find the permutation $p^{*}$ and hide $p^{*}(1), p^{*}(2), p^{*}(3)$ and $p^{*}(4)$ in the first eight coefficients.

- Transform the binary sequence of the message $m$ into $m^{\prime}$ using $p^{*}$ and $f$ and hide it in the coefficients starting from the seventeenth one.

- Apply the inverse Faber-Schauder discrete wavelet transform to obtain the stego image.

\section{Extraction algorithm}

- Read the stego image as two dimensional file.

- Apply the Faber-Schauder DWT to the stego image.

- Extract the permutation $p^{*}$ from the first eight coefficients and the identifier of the the key $\sigma$ from the second eight coefficients.

- Extract the binary sequence $m^{\prime}$ from the coefficients, and reconstruct $m$ using the function $f$ and permutation $p^{*}$.

- Regroup the binary sequence $m$ by blocks of 8 bits to obtain the hidden message.

\section{EXPERIMENTAL RESULTS AND DISCUSSION}

Experiments were accomplished to assess the performance of the proposed method using a variety of $512 \times 512$ grayscale images of the SIPI database, containing some images which are frequently utilized in tests, like "Baboon", "Peppers", "Lena" and "Elaine" (see Fig. 2).

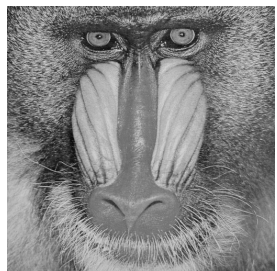

Baboon

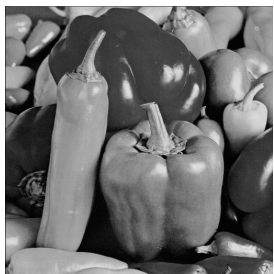

Peppers

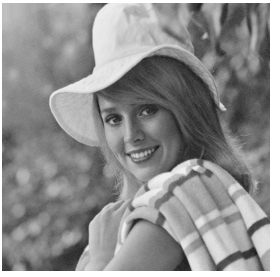

Elaine

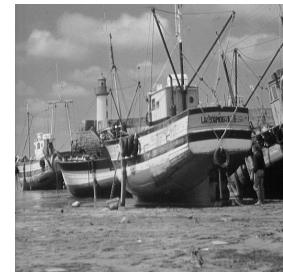

Boat

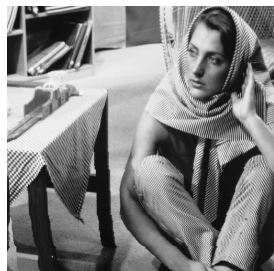

Barbara

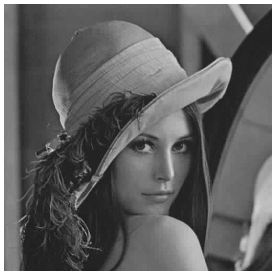

Lena

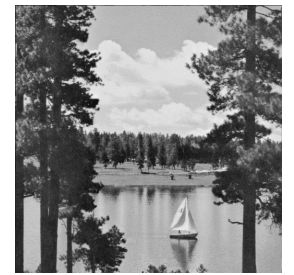

Lake

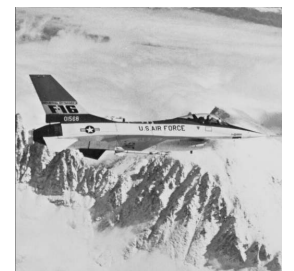

F16

Figure 2. Some of the images used in the experiment

The proposed work is compared with the methods developed by Amin [13], Miri [16] and Al-Dmour [17]. The test of the proposed work and the comparison are based on the following metrics [18]: 


$$
\begin{aligned}
& P S N R=10 \log \left(\frac{255^{2}}{M S E}\right) \quad ; N A E=\frac{\sum_{i=0}^{M-1} \sum_{j=0}^{N-1}|S(i, j)-C(i, j)|}{\sum_{i=0}^{M-1} \sum_{j=1}^{N} C(i, j)} \\
& \sum_{i=0}^{M-1} \sum_{j=0}^{N-1}(S(i, j)-C(i, j))^{2} \\
& \sum_{i=0}^{M-1} \sum_{j=0}^{N-1} C(i, j)^{2}
\end{aligned} \quad \text { NCC }=\frac{\sum_{i=1}^{N} \sum_{j=1}^{N}\left(C(i, j)-\mu_{C}\right)\left(S(i, j)-\mu_{S}\right)}{\sqrt{\sum_{i=1}^{M} \sum_{j=1}^{N}\left(C(i, j)-\mu_{C}\right)^{2}} \sqrt{\sum_{i=1}^{M} \sum_{j=1}^{N}\left(S(i, j)-\mu_{S}\right)^{2}}}
$$

The PSNR is the Peak Signal to Noise Ratio, it is calculated using the MSE. The more PSNR increases, the more the steganographic scheme is imperceptible. The $N A E$ is the Normal Absolute Error, it measures the absolute value of the error between the cover and stego images. Small values of $N A E$ (close to 0) are a sign of good imperceptibility. $I F$ is the Image Fidelity, the quantity $1-I F$ measures the ratio of the energy of the error between the cover and stego images to the energy of the cover image. Obviously, good imperceptibility requires that $1-I F$ is very close to 0 , which means that $I F$ has to be very close to 1 . The Normalized Correlation Coefficient NCC is a scalar product of the normalized vectors $v_{C}$ and $v_{S}$ while $v_{C}$ is the cover image minus its mean value $\mu_{C}$ and $v_{S}$ is the stego image minus its mean value $\mu_{S}$, so it takes values between -1 and 1 . The closer NCC is to 1 , the more similar are the images. If it is close to 0 , the images are uncorrelated, and if it is close to -1 , the images are said opposite.

\subsection{Test of the proposed method}

To test the proposed method, we used a set of 100 images with different modalities, downloaded from the SIPI image database.

Table 1. Imperceptibility for the proposed method

\begin{tabular}{lcccccccc}
\hline Metrics & PSNR & NAE & IF & NCC & PSNR & NAE & IF & NCC \\
\hline Data & \multicolumn{9}{c}{3000 bytes } \\
\hline Min & 61.55 & $2.01 \mathrm{e}-4$ & 0.999973 & 0.999654 & 58.53 & $4.01 \mathrm{e}-4$ & 0.999964 & 0.999371 \\
Max & 62.31 & $1.38 \mathrm{e}-3$ & 0.999999 & 0.999997 & 59.25 & $2.77 \mathrm{e}-3$ & 0.999998 & 0.999994 \\
Mean & 61.71 & $3.69 \mathrm{e}-4$ & 0.999997 & 0.999948 & 58.68 & $7.42 \mathrm{e}-4$ & 0.999994 & 0.999893 \\
\hline Data & \multicolumn{7}{c}{12000 bytes } \\
\hline Min & 56.76 & $6.02 \mathrm{e}-4$ & 0.999921 & 0.999073 & 55.52 & $8.05 \mathrm{e}-4$ & 0.999893 & 0.998526 \\
Max & 57.53 & $4.14 \mathrm{e}-3$ & 0.999997 & 0.999987 & 56.25 & $5.51 \mathrm{e}-3$ & 0.999996 & 0.999982 \\
Mean & 56.92 & $1.11 \mathrm{e}-3$ & 0.999991 & 0.999842 & 55.67 & $1.48 \mathrm{e}-3$ & 0.999988 & 0.999778 \\
\hline Data & \multicolumn{9}{c}{18000 bytes } & & \multicolumn{5}{c}{24000 bytes } \\
\hline Min & 53.75 & $1.21 \mathrm{e}-3$ & 0.999841 & 0.998147 & 52.51 & $1.61 \mathrm{e}-3$ & 0.999787 & 0.997565 \\
Max & 54.49 & $8.29 \mathrm{e}-3$ & 0.999994 & 0.999972 & 53.28 & $1.11 \mathrm{e}-2$ & 0.999992 & 0.999962 \\
Mean & 53.91 & $2.22 \mathrm{e}-3$ & 0.999982 & 0.999671 & 52.65 & $2.96 \mathrm{e}-3$ & 0.999976 & 0.999554 \\
\hline
\end{tabular}

Table 1 presents the results of the imperceptibility test for the proposed method, based on the metrics PSNR, NAE, IF and NCC. In this simulation, we dissimulated in the 100 test images a text of 3, 6, 9, 12, 18 and 24 Kilo Bytes. The table gives the minimum, maximum and mean values. A steganography process is imperceptible when $P S N R$ is beyond $36 \mathrm{~dB}$. The PSNR values indicate a high level of imperceptibility, NAE values are very small, $N A E<10^{-2}$, and $I F$ is practically $1,|1-I F|<10^{-4}$. NCC values are very close to 1 , $|1-N C C|<10^{-3}$, which proves that the cover and stego images are practically identical. Figure 3 exhibits the evolution of the PSNR for all the test images as the size of the hidden data increases. The PSNR diminishes, because when we hide larger data, the error becomes important. However, the drop of the imperceptibility becomes slower, when data size increases from 12 Kilo to 18 Kilo and from 18 Kilo to 24 Kilo, the mean PSNR decreases by $1.76 \mathrm{~dB}$ and $1.26 \mathrm{~dB}$ respectively. 


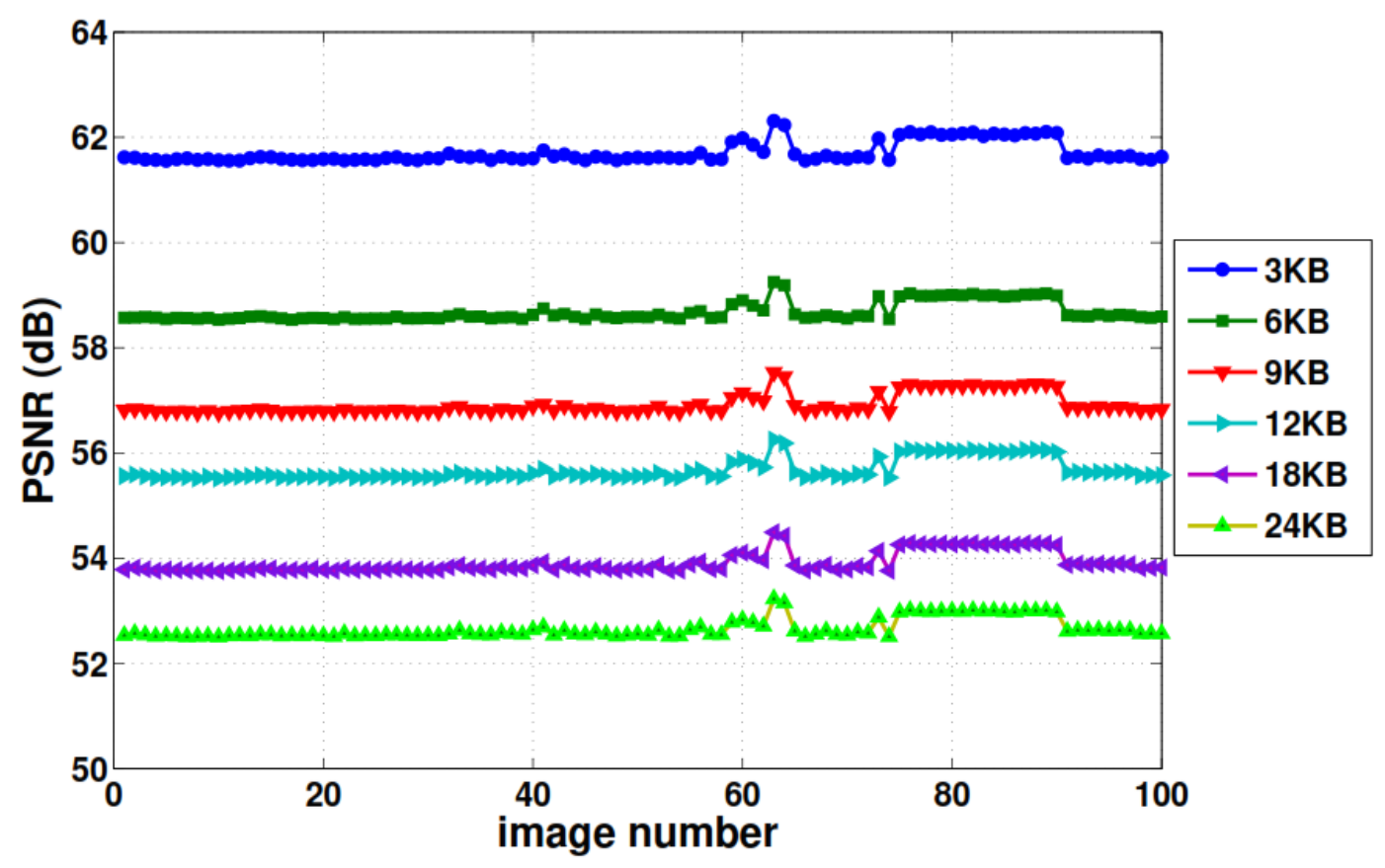

Figure 3. Capacity-Imperceptibility

\subsection{Comparison to literature}

The proposed work is compared to the methods developed by Amin [13], Miri [16] and Al-Dmour [17]. The tests of the comparison respects the same conditions (images, size of hidden data) utilized in these works.

Table 2 shows the results of comparison of PSNR to Amin's work for the four images used in his work, and table 3 compares the capacity of hiding. The proposed work provides a larger capacity $\frac{3}{4} M N-8$ bits, about 2.3 times the one of Amin, the subtracted 8 bits are reserved to hide the permutation $p^{*}$. In the algorithm he proposed, Amin does not hide data in all the wavelet coefficients, he selects the location where to hide data via the zero tree method, hence the capacity is diminished. On another hand, even concerning the imperceptibility, the proposed work still has better results, for 100,500 and $1 K$ bytes, the difference is approximatively $1 \mathrm{~dB}$. But, when we hide $5 K, 10 K$ and $15 K$ bytes, the difference becomes $3 \mathrm{~dB}$.

Table 2. Comparison of PSNR to Amin [13]

\begin{tabular}{llcccccc}
\hline Image & Method & 100 & 500 & 1000 & 5000 & 10000 & 15000 \\
\hline \multirow{2}{*}{ Barbara } & Amin & 73.98 & 66.61 & 63.64 & 65.55 & 53.64 & 52.02 \\
& Proposed & 76.57 & 69.46 & 66.38 & 59.37 & 56.35 & 54.59 \\
Peppers & Amin & 74.12 & 66.61 & 63.78 & 56.54 & 53.58 & 51.89 \\
& Proposed & 76.61 & 69.45 & 66.42 & 59.34 & 56.34 & 54.57 \\
Baboon & Amin & 75.62 & 68.18 & 62.89 & 56.06 & 53.32 & 51.75 \\
& Proposed & 76.59 & 69.40 & 66.38 & 59.35 & 56.32 & 54.56 \\
Lena & Amin & 73.58 & 66.07 & 63.01 & 56.18 & 53.38 & 51.65 \\
& Proposed & 76.57 & 69.42 & 66.41 & 59.37 & 56.37 & 54.59 \\
\hline
\end{tabular}

Table 4 shows the results of the comparison of the PSNR to Miri [16] and Al-Dmour [17], we respected the size of hidden data used in [16]. The proposed work has higher values. For Miri, the difference is around $3.6 \mathrm{~dB}$. In fact, Miri may hide data in more than one bit on a wavelet coefficient depending on the weight (position) of the most significant bit, the greater is the position, the more bits of the coefficients are used to embed data, in this case, the error generated from the dissimulation increases, which affected his PSNR values. As for Al-Dmour [17], the difference starts with $3 d B$, authors hide data in the edge coefficients and use the $X O R$ cording in order to minimize the error of the dissimulation. However, as the size of data increased, more 
Table 3. Comparison of the hiding capacity to Amin [13]

\begin{tabular}{lccc}
\hline Image & Size & Amin [13] & Proposed \\
\hline Lena & $128 \times 128$ & 5145 & 11891 \\
Lena & $256 \times 256$ & 20622 & 48371 \\
Lena & $512 \times 512$ & 82578 & 195059 \\
Peppers & $128 \times 128$ & 5223 & 11891 \\
Peppers & $256 \times 256$ & 20694 & 48371 \\
Peppers & $512 \times 512$ & 83846 & 195059 \\
\hline
\end{tabular}

Table 4. Comparison of PSNR to Miri [16] and Al-Dmour [17].

\begin{tabular}{lccr}
\hline Data size & Al-Dmour & Miri & Proposed \\
\hline 6300 bits & 64.76 & 63.80 & 67.44 \\
12800 bits & 61.50 & 60.66 & 64.32 \\
28800 bits & 56.91 & 56.79 & 60.78 \\
51200 bits & 52.62 & 54.78 & 58.28 \\
67700 bits & 50.28 & 53.68 & 57.06 \\
\hline
\end{tabular}

bits of the edge coefficients are used to dissimulate data (and depending of the cover image complexity, more bits of the coefficient may be used), which decreases significantly the PSNR. Hence, the difference enlarged to about $7 \mathrm{~dB}$ since in our case, we use only one bit in each coefficient, and the optimal permutation $p^{*}$ transforms the message into the best match for the cover image.

\section{CONCLUSION}

In this paper, a steganographic method based on Faber-Schauder DWT is proposed. Data is divided into pairs of 2 bits, the same is done to the $L S B$ of the details in the transform domain. We establish a matrix that calculates the number of times where data and the coefficients are similar or opposite, and based on this matrix we find the permutation that transforms the message into the binary sequence that provides the most match possible to the coefficients $L S B$ s. Results showed good trade-off between capacity and imperceptibility, and higher values in both of them compared to existing methods. In our future works, we will study more how to minimize the error generated by the dissimulation and we will strengthen the security through the analysis of the hiding's effect on the histogram.

\section{REFERENCES}

[1] C. K. Chan and L. M. Cheng, "Hiding data in images by simple LSB substitution," Pattern Recognition, vol. 37, pp. 469-474, 2004.

[2] C. C. Chang, J. Y. Hsiao and C. S. Chan, ”Finding optimal least-significant-bit substitution in image hiding by dynamic programming strategy," Pattern Recognition, vol.36, pp. 1583-95, 2003.

[3] E. Alrashed, S. S. Alroomi, "Hungarian-Puzzled Text with Dynamic Quadratic Embedding Steganography," International Journal of Electrical and Computer Engineering (IJECE), vol. 7, pp. 799-809, 2017.

[4] A. Benhfid, E. B. Ameur and Y. Taouil, "High capacity data hiding methods based on spline interpolation," 5th International Conference on Multimedia Computing and Systems (ICMCS), 2016, doi: 10.1109/ICMCS.2016.7905641.

[5] M. Tang, S. Zeng, X. Chen, J. Hu and Y. Du, "An adaptive image steganography using AMBTC compression and interpolation technique," International Journal for Light and Electron Optics, vol. 127, pp. 471-477, 2016.

[6] J. Hu and T. Li, "Reversible steganography using extended image interpolation technique," Computers \& Electrical Engineering, vol. 46, pp. 447-455, 2015.

[7] M. B. Jahromi and K. Faez, ”An Adaptive Steganography Scheme Based on Visual Quality and Embedding Capacity Improvement", International Journal of Electrical and Computer Engineering, vol. 4, pp. 573-584, Aug 2014.

[8] M. Hussain, A. W. Abdul Wahab, A. T. S. Ho, N. Javed and K.H. Jung, "A data hiding scheme using parity-bit pixel value differencing and improved rightmost digit replacement," Signal Processing: Image 
Communication, vol. 50, pp. 44-57, 2017.

[9] M. S. Arya, M. Rani and C. S. Bedi, "Improved Capacity Image Steganography Algorithm using 16Pixel Differencing with n-bit LSB Substitution for RGB Images," International Journal of Electrical and Computer Engineering", vol. 6, pp. 2735-2741, Dec 2016.

[10] S. Khan, T. Bianchi, "Ant Colony Optimization (ACO) based Data Hiding in Image Complex Region," International Journal of Electrical and Computer Engineering (IJECE), Vol. 8, pp. 379-389, Feb 2018.

[11] K. Wang, Z. M. Lub and Y. J. Hu, "A high capacity lossless data hiding scheme for JPEG images," Journal of Systems and Software, vol. 86, pp. 1965-1975, 2013.

[12] K. Qazanfari and R. Safabakhsh, "A new steganography method which preserves histogram: Generalization of LSB++," Information Sciences, vol. 277, pp. 90-101, 2014.

[13] S. A Seyyedi and N. Ivanov, "A Novel Secure Steganographic Method Based on Zero Tree Method," International Journal of Advanced Studies in Computer Science \& Engineering, vol. 3, no. 3, 2014.

[14] Y. Taouil, E. B. Ameur, M. T. Belghiti, "New Image Steganography Method Based on Haar Discrete Wavelet Transform". EMENA-TSSL, Advances in Intelligent Systems and Computing, vol. 520, pp. 287297, Oct 2016.

[15] Y. Taouil, E. B. Ameur, A. Benhfid, R. Harba and R. Jennane, "A Data Hiding Scheme Based on the Haar Discrete Wavelet Transform and the K-LSB," International Journal of Imaging and Robotics, vol. 17, pp. 41-53, 2017.

[16] A. Miri and K. Faez, "An image steganography method based on integer wavelet transform," Multimed Tools Appl, 2017, doi:10.1007/s11042-017-4935-z

[17] H. Al-Dmour and A. Al-Ani, "A steganography embedding method based on edge identification and XOR coding," Expert Syst Appl, vol. 46, pp. 293306, 2016.

[18] M. S. Subhedar and V. H. Mankar, "Image steganography using redundant discrete wavelet transform and QR factorization," Computers and Electrical Engineering, vol. 54, pp. 406-422, 2016.

\section{BIOGRAPHIES OF AUTHORS}

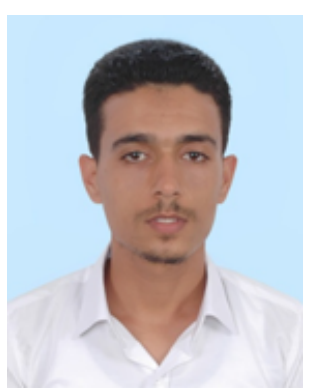

Youssef Taouil is a PhD Student at the faculty of sciences in Ibn Tofail University, he obtained the Engineering diploma in electronics and embedded systems from the national school of applied sciences at the same University (2014). His researches are focused on steganography and data hiding.

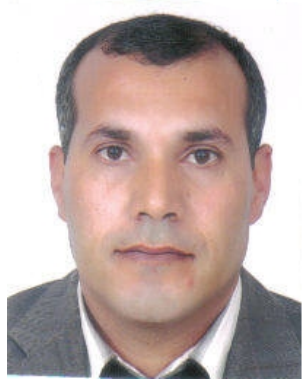

El Bachir Ameur is a full Professor of computer sciences at the University of IbnTofail, Faculty of science, Kenitra (Morocco), where he is affiliated to the LaRIT Laboratory. In 2002 he received the Ph. D. degree in numerical analysis and computer sciences from the University of Mohamed I Oujda (Morocco). His Ph. D. concerned approximation and reconstruction of 2D/3D data by spline and wavelet functions. His research interests concerns approximation and reconstruction of 2D/3D surfaces by spline and wavelets, signal and image processing, watermarking and steganography. 